cas seulement ont abouti à des écarts de lecture ayant dépassé $0,15 \%$ et ceci montre la valeur de la méthode proposée.

Institut Zootechnique de la Faculté de Médecine Vétérinaire de l'Université de Naples (Italie). Directeur : Professeur B. Ferrara.

BIBLIOGRAPHIE

J. C. Godfratn. Le Lait, 1956, 358, 492.

P. Schain. Science, 1949, 110, 121.

L. Gershenfield and B. I. UCKO-Milk and Food Technology, 1950, 13, 175.

L. Gentitini. Il Latte, 1950, 7, 219.

A. Salerno. Il Latle, 1953, 10, 553.

\title{
LA CONSERVATION PAR DIVERS ANTISEPTIQUES DES ÉCHANTILLONS DE LAIT DESTINÉS AUX ANALYSES (1)
}

\author{
par \\ Marie BEJAmbes, G. MOCQUOT et Paulette PAUThe \\ Station centrale de Microbiologie et Recherches laitières, Jouy-en-Josas
}

Les échantillons de lait destinés aux expertises chimiques officielles (notamment dans le cas où l'on soupçonne la fraude par mouillage ou écrémage), sont additionnés d'antiseptiques dont le rôle est d'assurer la conservation du produit depuis le moment du prélèvement jusqu'à celui de l'analyse. L'antiseptique le plus employé actuellement en France est le bichromate de potassium à la dose de 1 gramme par litre de lait. Or, l'expérience a montré que son action n'est pas toujours suffisante : les échantillons présentent trop souvent, au moment de l'analyse, les signes d'une altération plus ou moins avancée.

Dans ces conditions, l'analyse ne permet pas de se faire une idée exacte de la composition qu'avait le lait lors de son prélèvement et il s'ensuit que les conclusions de l'expert, s'il peut en formuler, risquent d'être incomplètes et même, dans certains cas, erronées.

L'emploi du bichromate a toujours été l'objet de vives critiques. Dès 1912, RocQues [1] avait constaté que son action n'est efficace que si le lait traité n'est pas acide. Sinon, l'acide lactique réduisant l'acide chromique, véritable agent de la conservation, les bactéries ne sont plus entravées dans leur développement.

Kling, Gelin et Lassieur, [2] d'une part, Magnier de la Source [3] d'autre part, ont attiré l'attention sur le mauvais état des laits ainsi traités. Hinard [4] a étudié la répercussion de l'alté-

(1) Annales de Technologie (I.N.R.A.), 1956, n 4, 539. 
ration de ces laits sur les résultats de l'analyse, A son avis, l'addition de bichromate ne fait que retarder le développement microbien. Il estime également qu'il agit plus énergiquement sur les ferments lactiques que sur les ferments protéolytiques, mais que, suivant les circonstances, cette action sélective se manifeste plus ou moins activement.

L'action de l'antiseptique étant de courte durée, on pouvait envisager, lorsque son effet avait cessé de s'exercer pleinement, de faire agir un nouveau produit. C'est en partant de cette conception que Filaudeau [5], suivant une idée de Vitoux, a proposé l'emploi du formol et du trioxyméthylène. Letourneur, Hugon et VALin[6] étudiant ce procédé, ont conclu de façon formelle en sa faveur. Il est actuellement utilisé dans un certain nombre de services officiels.

D'autres antiseptiques ont été proposés: DenIGES [7] a conservé en parfait état pendant 10 ans des échantillons de lait additionnés de $1 \%$ d'une solution de phénol contenant 50 grammes de phénol dans 10 millilitres d'alcool. On a reproché à cette méthode d'opérer une dilution du lait. Fonzes-Diacon et Laforce [8] ont expérimenté un mélange à parties égales de bichromate et de trioxyméthylène.

Enfin, plus récemment, Vorret [9] a préconisé l'emploi de l'acide salicylique en solution amylique saturée (1 à $2 \mathrm{ml}$. dans $200 \mathrm{ml}$. de lait). A l'action de l'antiseptique il ajoute celle de la chaleur : les échantillons traités sont maintenus 6 à 12.heures à la température de $70^{\circ}$.

Il nous a paru intéressant de comparer l'action bactéricide de quel ques antiseptiques en suivant l'évolution de la flore microbienne d'échantillons de lait auxquels ces antiseptiques avaient été ajoutés. Nous avons étudié en même temps les variations de la teneur deces échantillons en constituants glucidiques et azotés qui sont parmi les plus utiles à connaître pour la détection des fraudes, la fraude par mouilláge en particulier.

Nos essais ont porté d'une part sur les antiseptiques utilisés par les Services officiels aux doses où ils sont généralement employés : bichromate de potassium à 1 gramme par litre ; formol et trioxyméthylène : 0 m. 6 d'une solution commerciale de formol à $50 \%$ et 4 pastilles de trioxyméthylène pesant chacune 0 gr. 400 environ par litre de lait.

Et nous avons étudié d'autre part l'action d'un composé organique du mercure le borate de phényl mercure (merfen), employé à la dose de 0 gr. 1 par litre $(20 \mathrm{ml}$. d'une solution aqueuse à $0,5 \%$ (1).

(1) Nous remerçions $M^{\text {me }}$ Ruiz à qui nous sommes redevables de l'échantill on de merfen qui nous a été nécessaire. 


\section{TECHNIQUE EXPÉRIMENTALE}

Des échantillons de 21.5 de lait cru dont la teneur en germes dépassait en moyenne 10 millions par millilitre étaient additionnés de l'antiseptique étudié et introduits dans des flacons stériles. Ils étaient maintenus pendant toute la durée de l'expérience à $30^{\circ}$, température considérée comme la plus favorable au développement de la plupart des germes microbiens du lait.

Immédiatement avant et après l'addition de l'antiseptique, puis à des intervalles de temps variables suivant les cas, on prélevait aseptiquement dans les flacons ainsi incubés les quantités de lait nécessaires pour effectuer le dénombrement des germes et les analyses chimiques.

D'autre part, un " essai témoin " a porté sur du lait cru, sans antiseptique, placé à l'étuve à $30^{\circ}$ pour lequel on a effectué les mêmes examens bactériologiques et chimiques que pour les laits additionnés d'antiseptiques.

\section{A. Examens bactériologiques}

On a déterminé :

a) le nombre total de germes cultivant sur boîte de Petri en milieu gélosé à base de lait digéré par la papaïne (GuItTonneau et Chevalier) [10].

b) le nombre de germes cultivant en gélose profonde sur le milieu de Hirsch et Grinsted (R. C. M.) [11]. L'anaérobiose était réalisée suivant la technique indiquée par Dorner et al. [12].

\section{B. Analyses chimiques}

Elles ont comporté :

a) la détermination de l'acidité, et, dans quelques cas, du $p \mathrm{H}$.

b) le dosage du lactose; celui-ci a été dosé après hydrolyse totale. On sait en effet que l'action de certains microorganismes provoque rapidement une hydrolyse partielle du lactose, ce qui se traduit par une augmentation du pouvoir réducteur et peut masquer la disparition par fermentation d'une quantité de lactose appréciable. Le dosage des sucres réducteurs après hydrolyse totale permet d'éliminer cette cause d'erreur. Nous avons adopté la technique préconisée par SaEnz Lascano RuIz [13]. Les résultats sont exprimés en lactose hydraté.

c) Dosage des substances azotées.

Nous avons dosé l'azote total, l'azote du filtrat obtenu après précipitation de la caséine et l'azote non protéique suivant la méthode de Rowland [14]. 


\section{RÉSULTATS}

\section{A. Lait sans antiseptique}

Un lait contenant 6.000 germes par millilitre a été porté à $30^{\circ}$ et les prélèvements ont été effectués immédiatement, puis 2 h. 30,5 h., 7 h. 30 plus tard. Jusqu'à ce moment, les modifications chimiques se sont révélées très faibles, malgré un développement important de la flore microbienne. Après un séjour de 17 heures environ au frigorifique, à la température de $5^{\circ}$, le lait a été ramené à $30^{\circ}$ et des prélèvements ont de nouveau été effectués jusqu'au moment où s'est manifesté un début de coagulation du lait.

On trouvera sur le tableau I les conditions de l'expérience et les résultats des analyses effectuées.

TABLEAU I

LAIT GRU SANS ANTISEPTIQUE

\begin{tabular}{|c|c|c|c|c|c|c|c|}
\hline \multicolumn{2}{|c|}{ Incubation } & \multirow{2}{*}{$\begin{array}{l}\text { Acidité } \\
\text { degrés } \\
\text { Dornic }\end{array}$} & \multicolumn{2}{|c|}{ Lactose } & \multicolumn{2}{|c|}{$\mathrm{N}$ non protéique } & \multirow{2}{*}{$\begin{array}{c}\text { Germes totaux } \\
\text { (aérobies } \\
\text { par ml. }\end{array}$} \\
\hline $\begin{array}{l}\text { Tempé- } \\
\text { rature }\end{array}$ & Durée & & mgr./ml. & $\begin{array}{l}\text { var. \% } \\
(a)\end{array}$ & $\mathrm{mgr} / \mathrm{ml}$. & $\begin{array}{l}\text { var. \% } \\
\text { (b) }\end{array}$ & \\
\hline \multirow{4}{*}{$+30^{\circ}$} & 0 & 20 & 47 & & 0,32 & & $6 \times 10^{3}$ \\
\hline & 2 h. 30 & 21 & 47 & & 0,33 & & $165 \times 10^{3}$ \\
\hline & $5 \mathrm{~h}$. & 21 & 47 & & 0,33 & & $4.800 \times 10^{3}$ \\
\hline & 7 h. 30 & 21 & 46,8 & 0,4 & 0,33 & & $14 \times 10^{8}$ \\
\hline$+5^{\circ}$ & $24 \mathrm{~h}$. & 25 & 46,1 & 1,9 & 0,33 & & $126 \times 16^{0}$ \\
\hline \multirow{2}{*}{$+30^{\circ}$} & 26 h. 30 & 34 & 45,1 & 4,0 & 0,35 & 9,3 & $500 \times 10^{8}$ \\
\hline & 29 h. 15 & 73 & 41,7 & 11,2 & 0,40 & 25 & $750 \times 10^{8}$ \\
\hline
\end{tabular}

(a) Diminution en pourcentage de la valeur initiale.

(b) Augmentation en pourcentage de la valeur initiale.

\section{B. Examens bactériologiques des laits additionnés d'antiseptiques}

a) Observation préliminaire

Le lait ou la dilution de lait ajouté au milieu gélosé au cours de la numération apporte, en même temps que les germes microbiens, une quantité d'antiseptique proportionnelle au volume de lait ensemencé. Or cette dose d'antiseptique peut être suffisante pour inhiber le développement microbien sur le milieu de culture. Il convient done tout d'abord de déterminer pour chaque antiseptique la " concentration-seuil " c'est-à-dire la concentration maxima dans 
le milieu gélosé au-dessus de laquelle l'antiseptique est susceptible d'exercer une action inhibitrice.

Le volume final de milieu ensemencé avec 1 millilitre de lait ou d'une dilution décimale de lait étant constant, on connaît sa concentration en antiseptique, soit $\mathrm{A}, \mathrm{A} \times 10^{1}, \mathrm{~A} \times 10^{2}$, etc...

Pour connaître la "concentration-seuil » il suffit de comparer à la numération des germes d'un lait cru. sur le milieu gélosé choisi (témoin), la numération des germes du même lait sur le même milieu additionné d'antiseptique à dose telle que la concentration finale dans la gélose soit $\mathrm{A}$ pour une première série, $\mathrm{A} \times 10^{1}$ pour une deuxième, $\mathrm{A} \times 10^{1}$ pour une deuxième, $\mathrm{A} \times 10^{2}$ pour une troisième, et ainsi de suite. On détermine ainsi les concentrations en antiseptique non inhibitrices. Parmi elles, la plus élevée est la "concentration-seuil ". Lorsqu'on la connaît il est facile de déterminer le volume de lait additionné d'antiseptique qui lui correcpond.

On a noté sur le tableau II le résultat de cette expérience pour les antiseptiques essayés.

Dans le cas du formol et du trioxyméthylène, il nous était difficile de tenir compte à la fois de l'influence de ces deux corps. En effet, leur action ne se manifeste pas en même temps : celle du formol se produit dès l'addition au lait de cet antiseptique. Le trioxyméthylène n'agit au contraire qu'au fur et à mesure de sa dissolution dans le lait. L'expérience nous ayant montré qu'une destruction massive des germes se produisait dans les premiers jours qui suivent l'addition des antiseptiques au lait, done pendant la période où l'action du formol est prédominante, nous nous sommes limités à la détermination de la "concentration-seuil" de ce corps.

Tableau II

ACTION INHIBITRICE DES ANTISEPTIQUES

SUR LE DEVELOPPEMENT DES GOLONIES MIGROBIENNES SUR MILIEU GÉLOSE

\begin{tabular}{|c|c|c|c|}
\hline Antiseptique & Bichromate de K & Formol & Merfen \\
\hline Concentration dans le lait...... & $10^{3}$ & $3 \times 10^{4}$ & $10^{4}$ \\
\hline $\begin{array}{c}\text { "Concentration-seuil " dans la } \\
\text { gélose } \ldots \ldots \ldots \ldots \ldots \ldots \ldots\end{array}$ & $10^{8}$ & $3 \times 10^{6}$ & $10^{7}$ \\
\hline Quantité de lait correspondant & & & \\
\hline à la " concentration-seuil " .. & $0,1 \mathrm{ml}$. & $0,1 \mathrm{ml}$. & $0,01 \mathrm{ml}$. \\
\hline
\end{tabular}

Au cours des numérations, nous n'avons donc pris en considération que les boîtes de Pétri ou les tubes ensemencés avec des quantités de Iait égales ou inférieures à celles ainsi déterminées. 
Arec des quantités de lait plus fortes, on doit admettre que le nombre de germes trouvé est inférieur au nombre de germes réellement présents dans le lait.

\section{b) Microbes aérobies}

L'examen bactériologique d'un certain nombre d'échantillons de lait additionnés d'antiseptiques nous a permis de faire les constatations suivantes :

Dans les laits bichromatés, le nombre de germes diminue rapidement, puis s'élève à nouveau. Ainsi, la teneur en germes d'un lait est passée de 2 milliards à mille par millilitres un jour après l'addition du bichromate. Puis les germes se sont multipliés à nouveau et 5 jours plus tard, le lait contenait 7 millions de microorganismes et était coagulé. L'action bactéricide du bichromate a donc été rapide mais 'de courte durée.

Le formol exerce une action plus prononcée. En effet, à partir du quatrième jour qui suit le traitement, les numérations microbiennes révèlent seulement la présence de quelques germes microbiens.

Quant au merfen, son action a été comparable à celle du formol bien qu'un peu plus lente. Là, encore, nous assistons à une diminution importante du nombre des microoganismes. Tous ne sont cependant pas détruits et il arrive, qu'après un temps plus ou moins long de conservation, on observe une nouvelle prolifération microbienne.

Nous avons porté sur le graphique I, les variations en fonction du temps des logarithmes du nombre de germes d'un même lait additionné des antiseptiques étudiés.

\section{c) Microbes se développant en anaérobiose}

Le nombre des microbes se développant en anaérobiose suit une évolution parallèle à celle des germes qui poussent sur la gélose èn boîtes de Petri. Ce fait est mis en évidence par le graphique II, où sont portées les variations en fonction du temps des logarithmes du nombre de germes par millilitre de lait trouvés dans les deux cas.

\section{Analyses chimiques des laits additionnés d'antiseptiques}

\section{a) Lait additionné de bichromate de potassium}

Le lait dont les résultats d'analyse ont été groupés dans le tableau III contenait au moment de la mise en expérience, 21 millions de germes par millilitres. Son acidité était de 19 degrés Dornic, sa teneur en azote de $5 \mathrm{mg} .58$ par millilitre. Au cours de cet essai, comme de ceux qui vont suivre, la teneur en azote n'a pas varié sensiblement. 


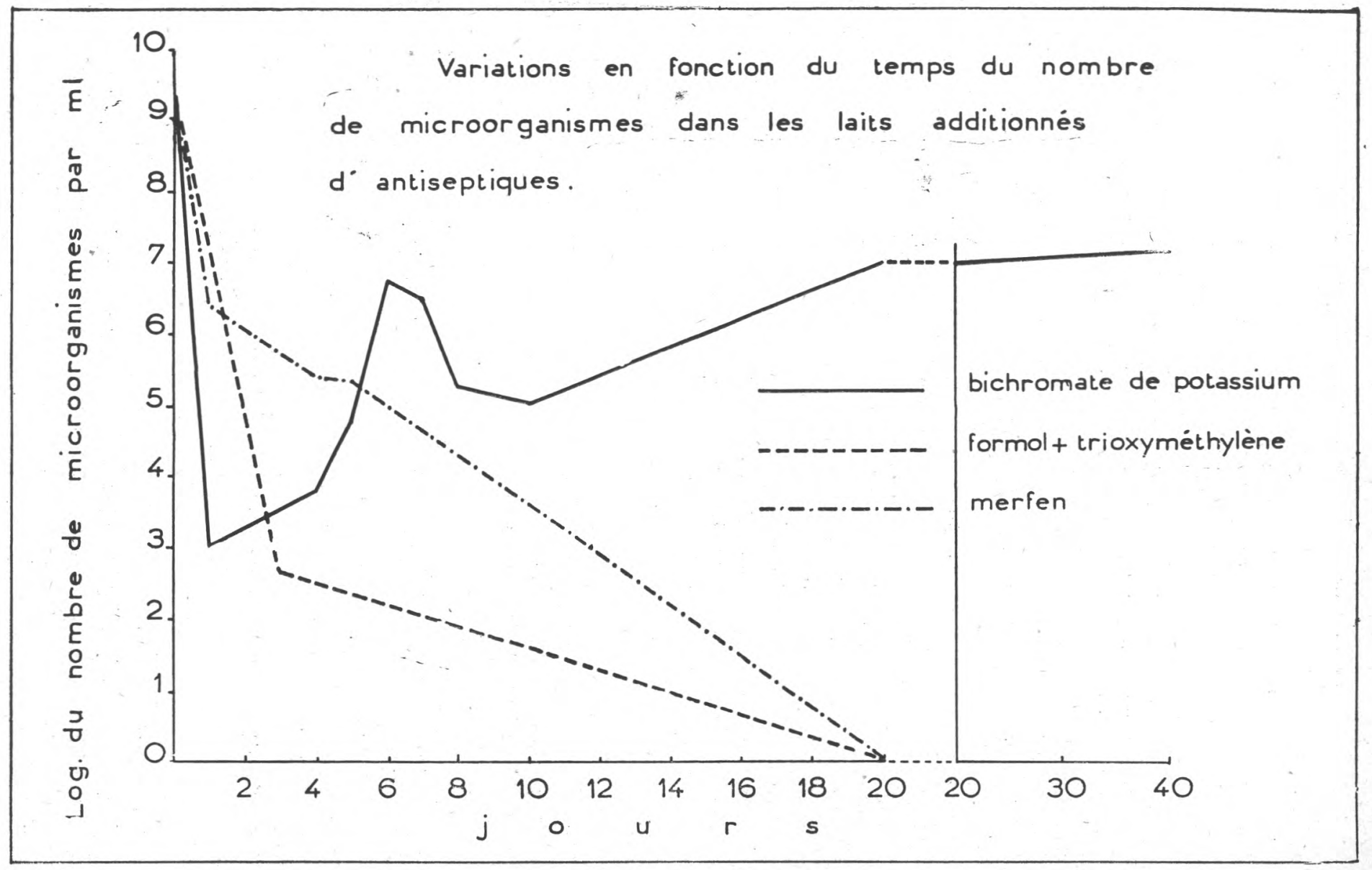

Graphique I 
Variation du nombre des mieroorganismes se développant au cours des 5 jours qui suivent l'addition des antiseptiques du lait
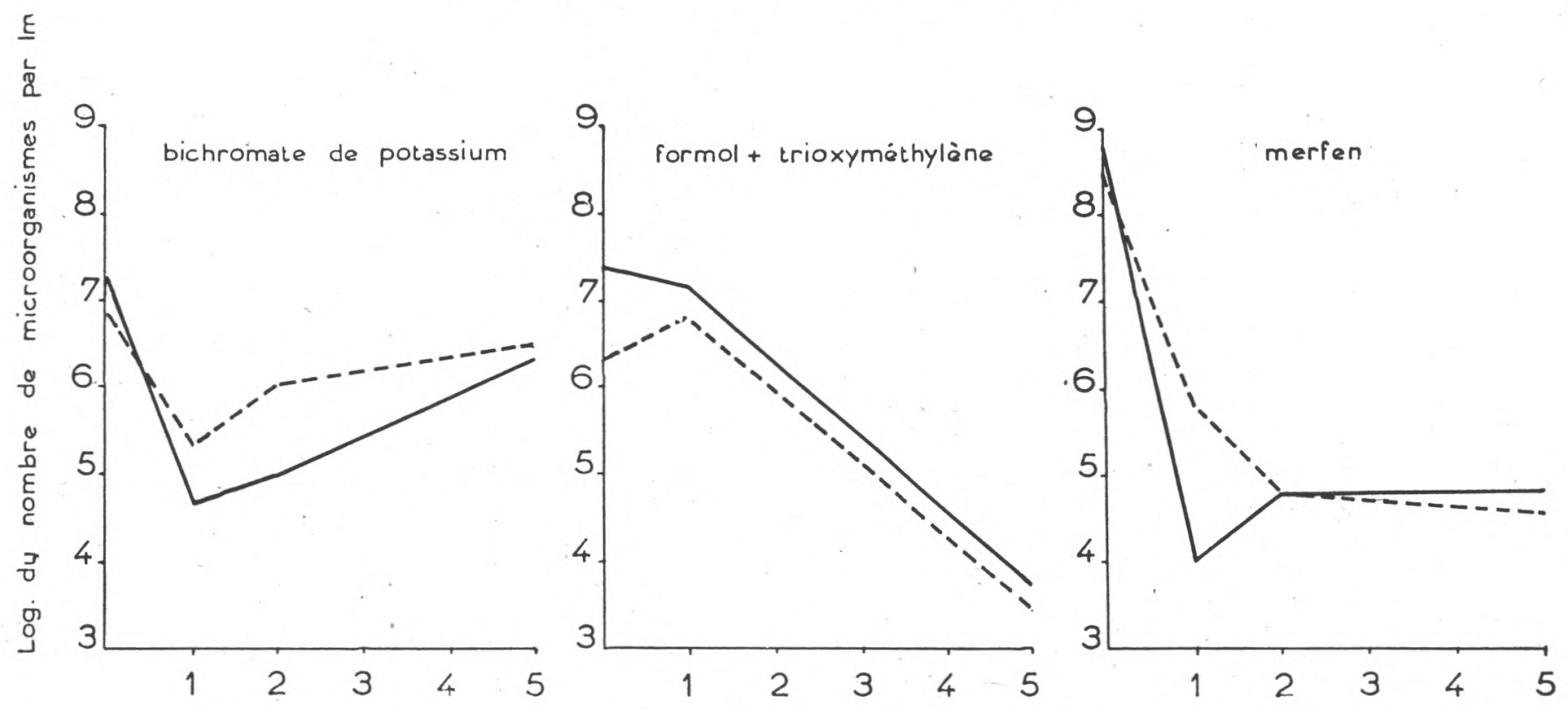

Microbes se développant en : aérobiose. , anaérobiose 
L'expérience a été arrêtée après 7 jours d'incubation à $30^{\circ}$, le lait étant alors coagulé.

On peut noter, après 7 jours, une diminution du lactose d'environ $10 \%$ et une augmentation de 60 à $70 \%$ de la fraction azotée non protéique. Ces résultats ont été confirmés au cours de plusieurs essais successifs.

TABLEAU III

LAIT ADDITIONNÉ DE BICHROMATE DE POTASSIUM

\begin{tabular}{|c|c|c|c|c|c|c|c|c|}
\hline \multirow[b]{2}{*}{ Date } & \multirow{2}{*}{$\begin{array}{l}\text { Nombre de jours } \\
\text { après l'addition } \\
\text { de l'antiseptique }\end{array}$} & \multicolumn{2}{|c|}{ Lactose } & \multicolumn{2}{|c|}{ N non caséine } & \multicolumn{2}{|c|}{$\mathrm{N}$ non protéique } & \multirow[b]{2}{*}{$p$ H } \\
\hline & & mgr./ml. & $\begin{array}{c}\text { var. } \% \\
(a)\end{array}$ & $\mathrm{mgr} . / \mathrm{ml}$. & $\begin{array}{c}\text { var. } \% \\
(b)\end{array}$ & mgr./ml. & $\begin{array}{c}\text { var. } \% \\
(b)\end{array}$ & \\
\hline $21 / 7$ & immédiatement avant & 48,4 & & 1,28 & & 0,31 & & 6,3 , \\
\hline $21 / 7$ & immédiatement après & 48,4 & & 1,27 & & 0,31 & & 6,3 \\
\hline $22 / 7$ & 1 & 46,2 & 4,5 & 1,34 & 4,5 & 0,31 & & 6,2 \\
\hline $23 / 7$ & 2 & 46,2 & 4,5 & 1,36 & 6,2 & 0,35 & 12,9 & 5,8 \\
\hline $26 / 7$ & 5 & 45,1 & 6,8 & 1,45 & 13,2 & 0,44 & 41,9 & 5,1 \\
\hline $27 / 7$ & 6 & 44,5 & 8,0 & 1,52 & 18,7 & 0,49 & 58,0 . & 5,1 \\
\hline $28 / 7$ & 7 & 42,7 & 9,8 & 1,60 & 25,0 & 0,52 & 67,7 & 4,7 \\
\hline
\end{tabular}

(a) Diminution en pourcentage de la valeur initiale.

(b) Augmentation en pourcentage de la valeur initiale.

TABLEaU IV

LAIT ADDITIONNÉ DE FORMOL ET DE TRIOXYMÉTHYLÈNE

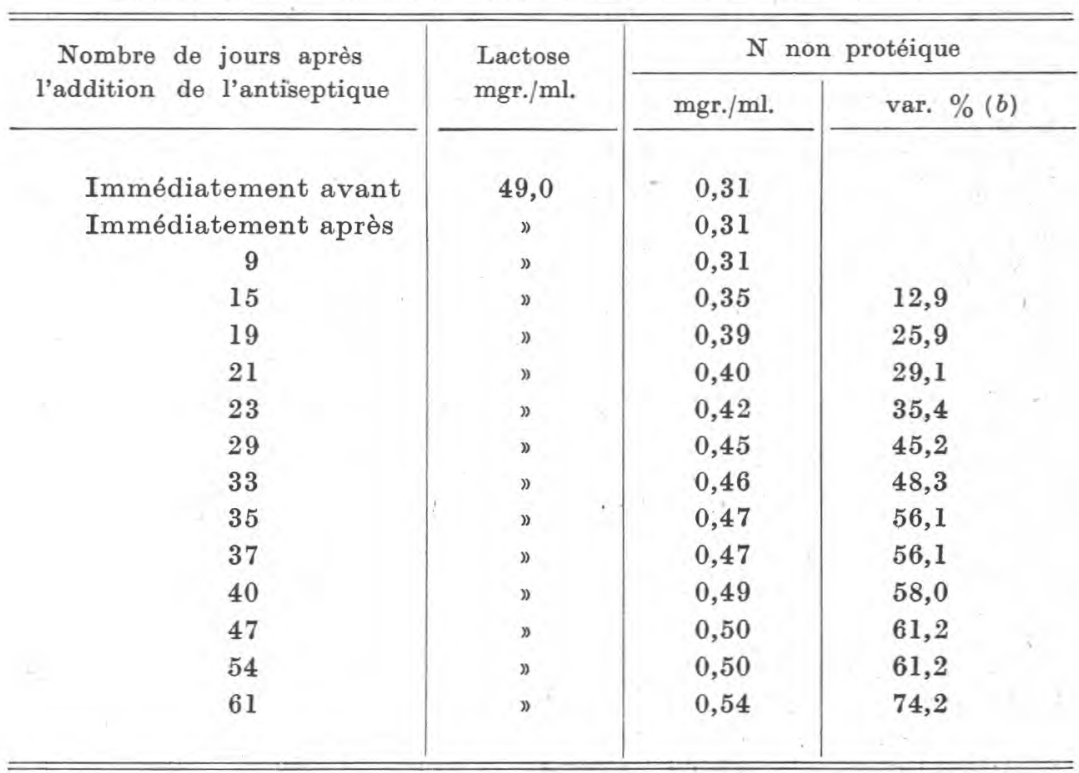

(b) Augmentation en pourcentage de la valeur initiale. 
Variations de $1^{\prime}$ azote non protéique et du lactose $d^{\prime} u n$ lait témoin.

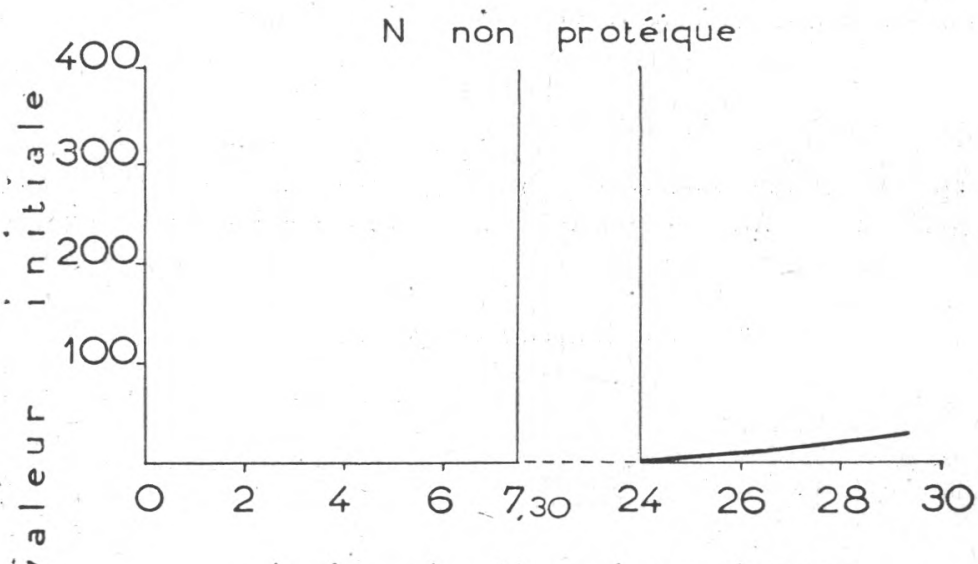

durée de $1^{\prime}$ essai en heures

$\begin{array}{llllllllll}0 & 0 & 2 & 4 & 6 & 7,30 & 24 & 26 & 28 & 30\end{array}$

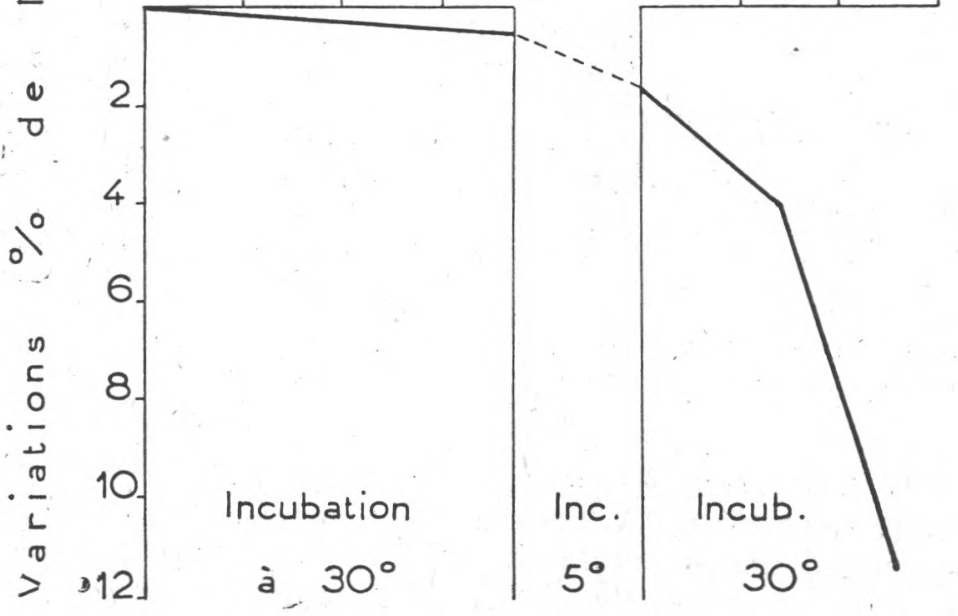

Lactose

Graphique III 


\section{b) Lait additionné de formol et de trioxyméthylène}

Le lait mis en expérience contenait à l'origine 35 millions de germes par millilitres. Le dosage de la caséine n'a pu être effectué, la présence du formol gênant rapidement sa précipitation à $p \mathrm{H} .4,6$ selon la technique de RowLAND.

Pendant les deux mois qu'à duré l'expérience, le taux de lactose est resté inchangé. Il y a eu par contre une augmentation appréciable de l'azote non protéique.

\section{c) Lait additionné de merfen}

Les résultats d'un essai portés sur le tableau $\mathrm{V}$ montrent, qu'après 15 jours environ à $30^{\circ}$, le lactose a faiblement diminué tandis que l'azote non protéique a considérablement augmenté.

\section{Tableau $\mathrm{V}$}

LAIT ADDITIONNÉ DE MERFEN

\begin{tabular}{|c|c|c|c|c|c|c|}
\hline \multirow{2}{*}{$\begin{array}{c}\text { Nombre de jours après } \\
\text { l'addition de l'antiseptique }\end{array}$} & \multicolumn{2}{|c|}{ Lactose } & \multicolumn{2}{|c|}{ N non caséine } & \multicolumn{2}{|c|}{$\mathrm{N}$ non protéique } \\
\hline & $\mathrm{mgr} . / \mathrm{ml}$ & $\begin{array}{l}\text { var. } \% \\
\text { (a) }\end{array}$ & $\mathrm{mgr} . / \mathrm{ml}$. & $\begin{array}{l}\text { var. } \% \\
(b)\end{array}$ & mgr. $/ \mathrm{ml}$. & $\begin{array}{c}\% \text { var. } \\
\text { (b) }\end{array}$ \\
\hline Immédiatement avant & 45,1 & & 1,02 & & 0,30 & \\
\hline Immédiatement après & 45,1 & & 1,01 & & 0,30 & \\
\hline 2 & 45,1 & & 1,20 & 17,7 & 0,47 & 56,7 \\
\hline 6 & 45,1 & & 1,75 & 71,6 & 0,77 & 156,7 \\
\hline 8 & 45,1 & & 1,84 & 80,4 & 0,91 & 203,3 \\
\hline 10 & 45,1 & & 1,96 & 92,1 & 1,05 & 250,0 \\
\hline 13 & 45,1 & & 2,17 & 112,7 & 1,19 & 296,7 \\
\hline 15 & 43,4 & 3,7 & 2,32 & 127,5 & 1,34 & 346,6 \\
\hline 20 & 43,4 & 3,7 & 2,42 & 137,2 & 1,48 & 393,4 \\
\hline 27 & & & 2,74 & 171,2 & 1,76 & 486,6 \\
\hline 34 & 43,3 & 4,0 & 2,92 & 186,2 & 2,08 & 593,3 \\
\hline
\end{tabular}

(a) Diminution en pourcentage de la valeur initiale.

(b) Augmentation en pourcentage de la valeur initiale.

A titre de comparaison, nous avons porté respectivement sur les graphiques III et IV les variations en fonction du temps de l'azote non protéique et du lactose d'un échantillon de lait sans antiseptique et de laits additionnés d'antiseptiques.

\section{d) Evolution de l'acidité des laits additionnés d'antiseptiques}

Nous avons suivi les variations de l'acidité et du taux de lactose d'un lait depuis le moment où il a été additionné le bichromate de potassium à la dose habituelle jusqu'à sa coagulation, le lait étant 
Variations de $1^{\circ}$ azote non protéique et du lactose $d^{\prime}$ échantillons de lait additionnés $d^{\prime}$ antiseptiques.
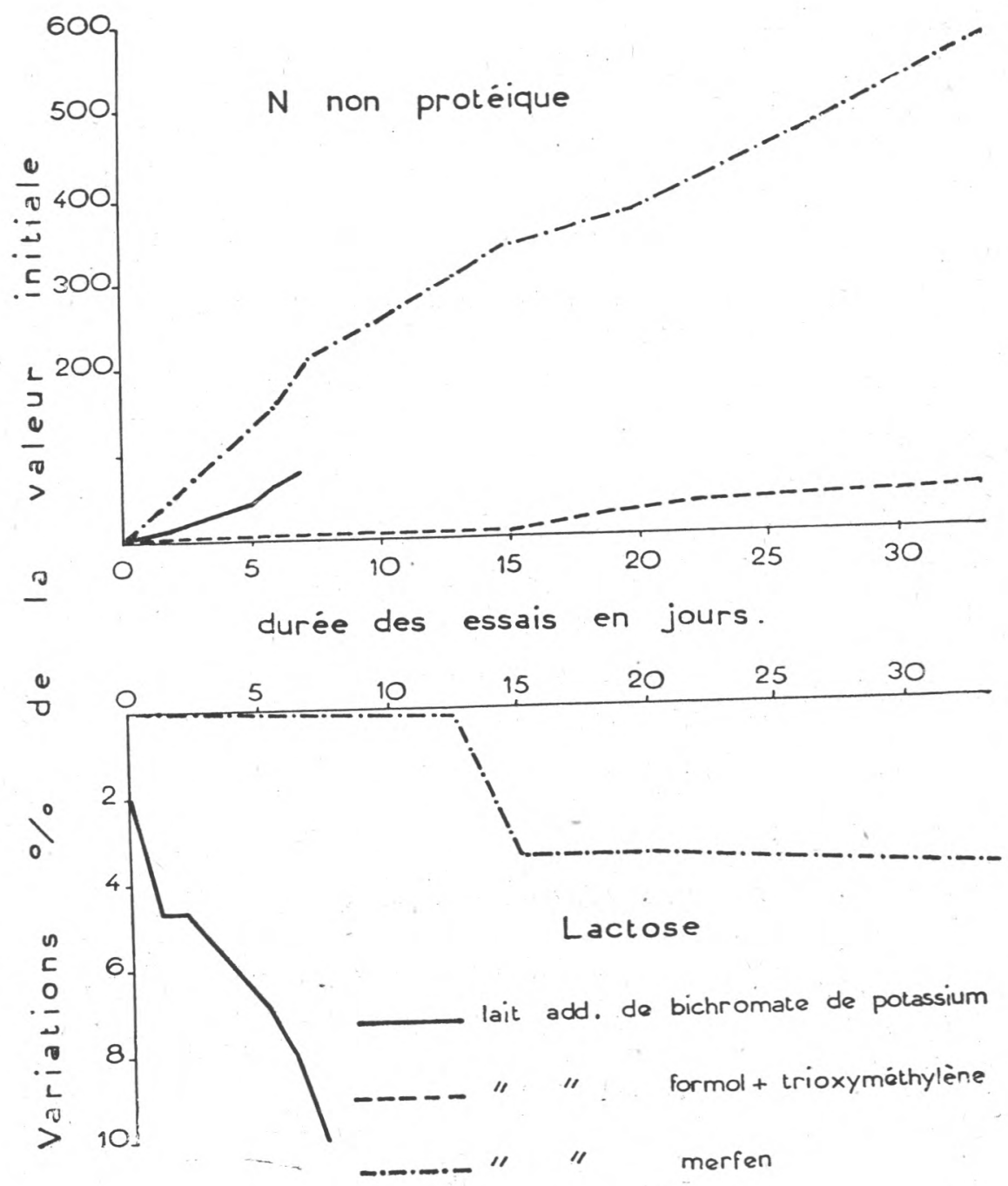

Graphique IV 
Tableat VI

VARIATIONS DE L'AGIDITÉ ET DU LAGTOSE D'UN LAIT ADDITIONNÉ DE BICHROMATE DE POTASSIUM

\begin{tabular}{|c|c|c|c|c|}
\hline \multirow{2}{*}{$\begin{array}{l}\text { Nombre de jours } \\
\text { après l'addition } \\
\text { de l'antiseptique }\end{array}$} & \multicolumn{2}{|c|}{$\begin{array}{c}\text { Acidité exprimée } \\
\text { en gramme } \\
\text { d'acide lactique par litre }\end{array}$} & \multicolumn{2}{|c|}{$\begin{array}{c}\text { Lactose } \\
\text { en gramme par litre }\end{array}$} \\
\hline & doséé & formée & dosée & disparu \\
\hline Immédiatement après & 3,3 & & 48,1 & \\
\hline 1 & 4,1 & 0,8 & 47,0 & 1,1 \\
\hline 3 & 6,0 & 2,7 & 44,8 & 3,3 \\
\hline 6 & 7,8 & 4,5 & 43,0 & 5,1 \\
\hline 7 & 8,2 & 4,9 & 42,5 & 5,6 \\
\hline
\end{tabular}

Variations de $1^{\circ}$ acidité et du lactose $d^{\circ}$ un

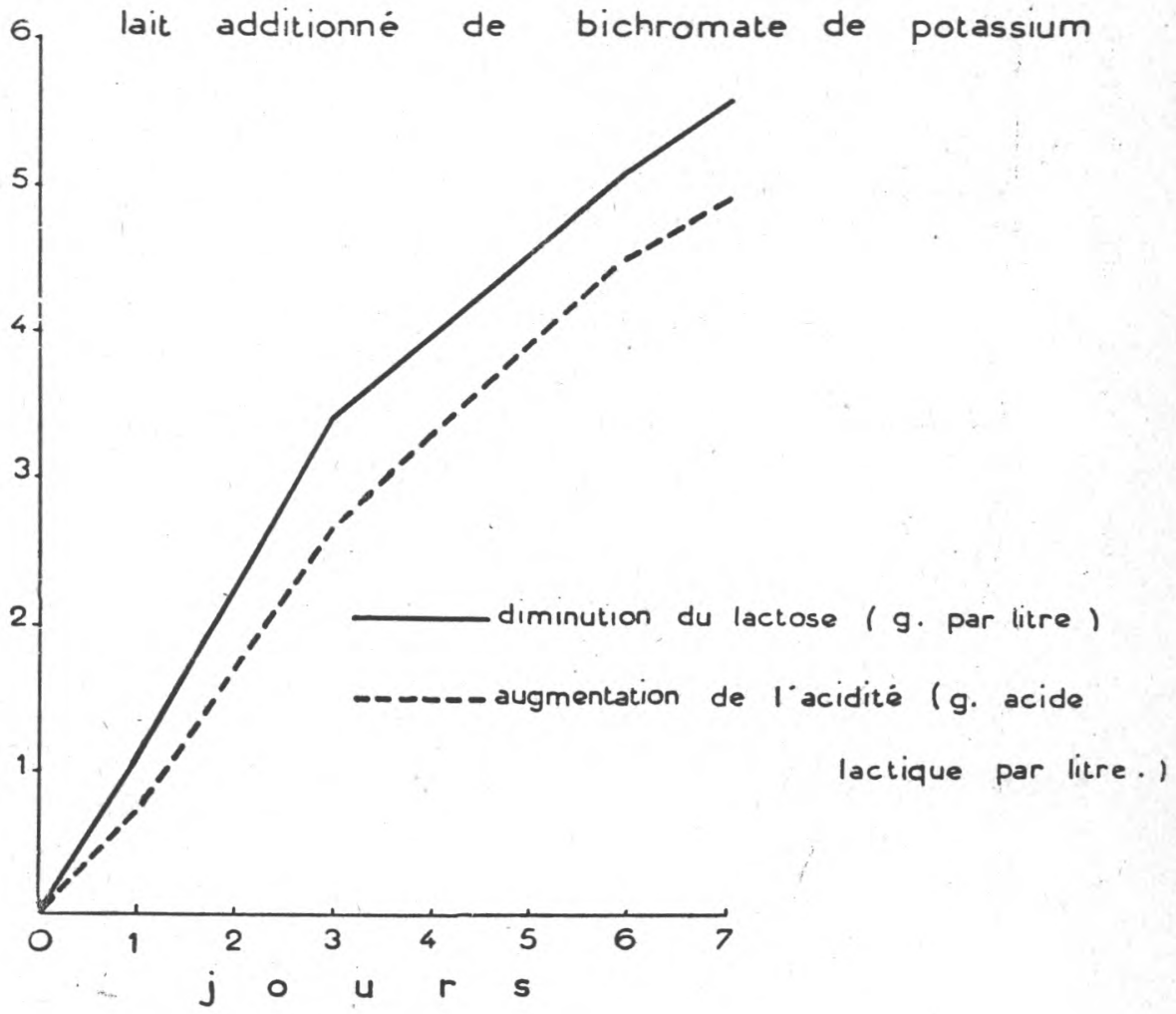

Graphique V 
Variations de $l^{\circ}$ ącidité $d^{\circ}$ un lait additionné de différents antiseptiques

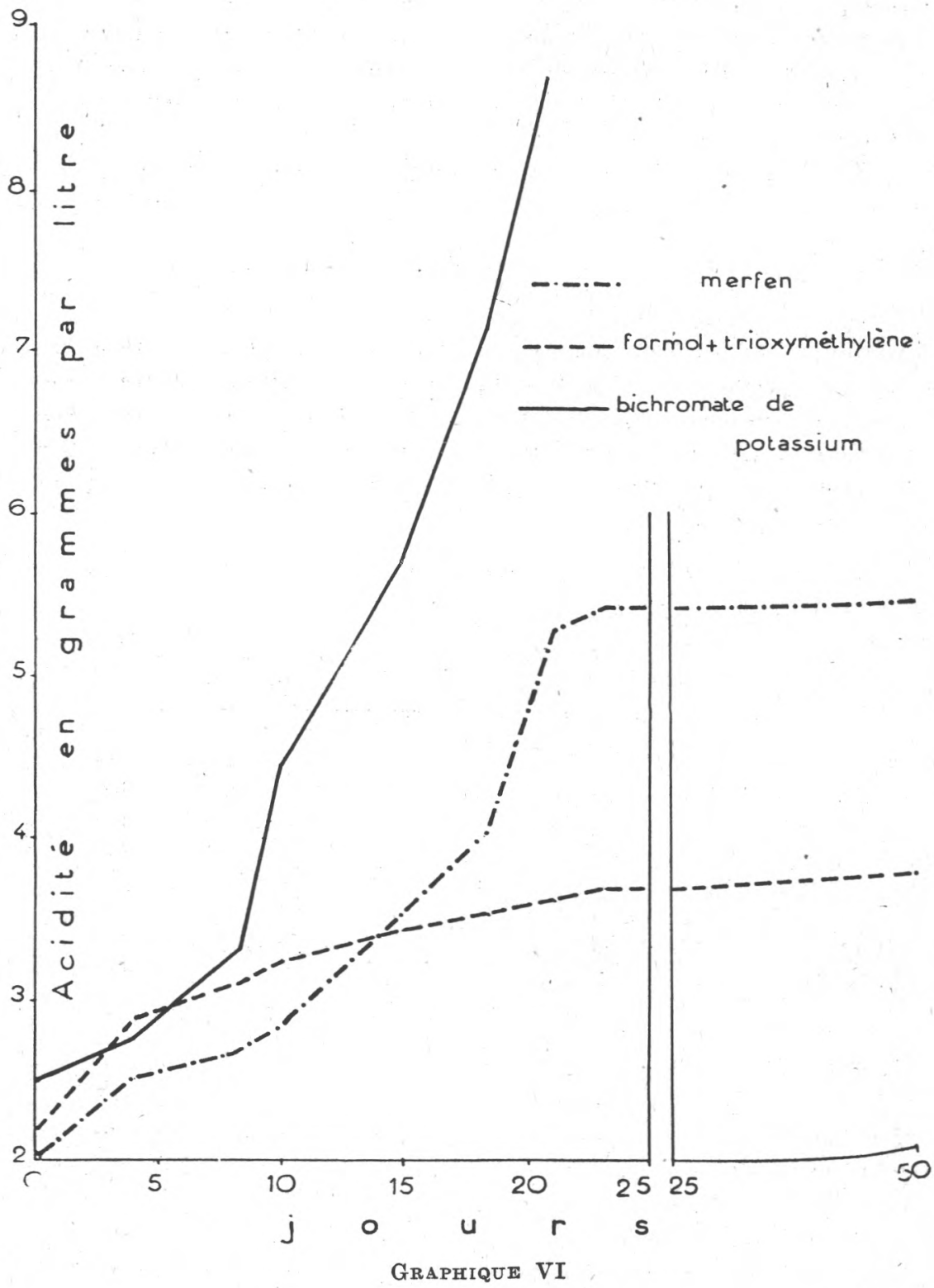


maintenu à $30^{\circ}$. Les résultats sont consignés dans le tableau VI et le graphique $\mathrm{V}$ où on voit la relation entre les quantités de lactose disparu et d'acide lactique formé.

Dans le cas du formol, on observe au début une acidification dont on peut rendre responsable la réaction entre le formol et les groupes aminés de la caséine. Ensuite, l'acidité reste constante. Nous avons vu plus haut que le taux de lactose ne variait pas.

Pour le lait additionné de merfen, l'acidité s'élève pendant les 20 premiers jours, puis reste constante.

Nous avons porté sur le graphique VI, les courbes de variations, en fonction du temps, de l'acidité des laits additionnés de l'un des trois antiseptiques étudiés.

\section{e) Protéolyse des laits additionnés d'antiseptiques}

Que le lait ait été ou non additionné d'antiseptique, nous avons toujours observé une protéolyse mesurable. Exception faite des laits auxquels on a ajouté formol et trioxvméthrlène, nous avons constaté également la disparition d'une partie du lactose. Mais l'intensité de ces deux phénomènes s'est révélée extrêmement différente suivant les cas.

TABLEaU VII

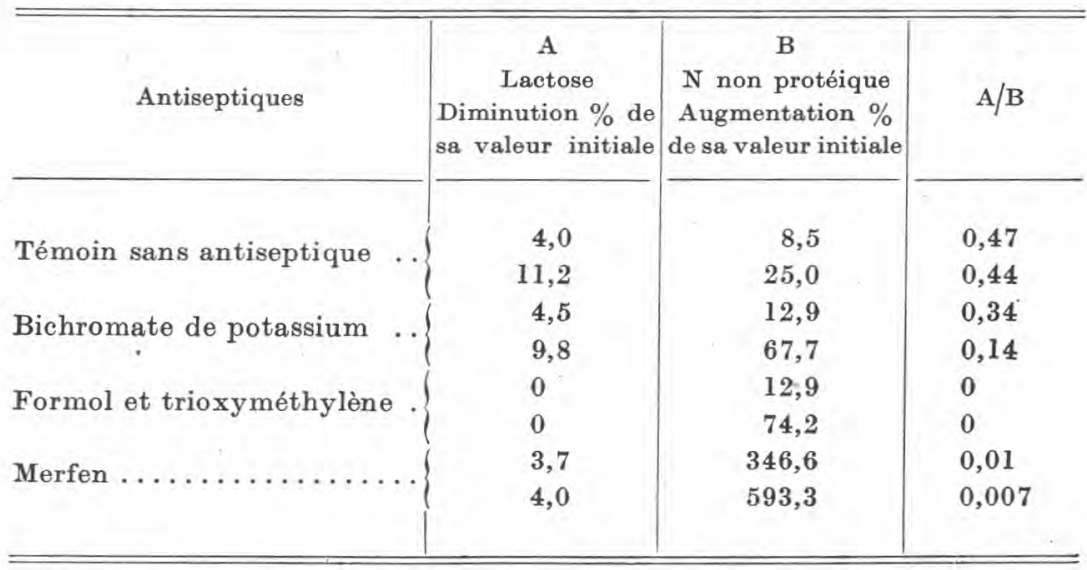

Pour illustrer ce fait, nous avons porté sur un même tableau (tableau VII), pour le lait témoin et pour les laits additionnés d'antiseptiques, les pourcentages d'augmentation de l'azote non protéique, les pourcentages de lactose disparu et enfin le rapport entre ces deux valeurs.

On voit que ce rapport est voisin de 0,4 dans le lait sans antiseptique mais diminue déjà sensiblement lorsque le lait est bichro- 
maté. Il atteint à peine 0,01 dans le lait additionné de merfen et est nul pour le lait formolé.

Nous nous sommes demandés si on ne pouvait pas attribuer à une action chimique indépendante de l'action des germes microbiens cette protéolyse des laits additionnés d'antiseptiques. Nous avons voulu vérifier cette hypothèse dans le cas où cette augmentation est particulièrement nette, celui du lait contenant du merfen.

Nous avons additionné de merfen à la dose habituelle $(0,01 \%)$ et à dose double $(0,02 \%)$ des échantillons de lait cru et des échantillons du même lait stérilisé (par chauffage à l'autoclave pendant 20 minutes à $118^{\circ}$ ).

47 jours après ces additions, (les échantillons ayant été maintenus à $30^{\circ}$ ) nous avons constaté que dans le lait stérilisé additionné de merfen à $0,01 \%$, l'azote non protéique avait augmenté de $10,1 \%$ et dans le lait ayant reçu double dose de merfen de $4,4 \%$. Dans les laits crus, les pourcentages d'augmentation correspondants étaient respectivement 166,3 et 978,4

Nous sommes done en droit de conclure que la protéolyse résultant de l'action propre de l'antiseptique ajouté au lait très est faible par rapport à celle que l'on observe avec du lait cru et que cette dernière résulte bien de la présence de microorganismes ou des enzymes qu'ils produisent.

Le fait que les microorganismes du lait cru additionné d'antiseptique ne sont plus capables de se développer — ou seulement en petit nombre - sur les milieux de culture semble en faveur d'une action enzymatique provenant des corps microbiens.

On doit noter cependant que la présence d'un antiseptique ne permet pas de compter avec certitude les germes présents lorsque ceux-ci sont peu nombreux, en particulier dans le cas du merfen pour lequel la protéolyse est aussi la plus marquée. Il est done possible que cette protéolyse soit due à un petit nombre de germes qui restent vivants et actifs mais qu'il n'est pas possible de déceler sur les milieux de culture avec la technique que nous avons suivie.

Nous avons vu d'autre part que la présence d'un antiseptique dans le lait inhibe moins la protéolyse que la dégradation du lactose. On peut penser que l'antiseptique agit soit en opérant une sélection des microorganismes soit en modifiant le système enzymatique des germes présents, chaque antiseptique possédant à cet égard une action qui lui est propre.

\section{CONCLUSION}

Aucun des antiseptiques essayés ne permet, à la dose où ils ont été utilisés, une stabilisation complète du lait.

Le bichromate n'évite ni la coagulation du lait ni sa protéolyse, 
ce qui complique le prélèvement d'un échantillon homogène, De plus le taux du lactose, dont la détermination est capitale dans la recherche des fraudes par mouillage, diminue rapidement. Le bichromate ne peut done pas être considéré comme un bon agent de conservation des échantillons de lait.

Le merfen, s'il freine considérablement la destruction du lactose, favorise par contre la protéolyse. Après un temps assez long, l'altération devient souvent visible à l'œil nu, le lait prenant un aspect particulier : une fine floculation se produit au sein d'un liquide translucide.

Dans les laits additionnés de formol et de trioxyméthylène, cette altération visible ne se manifeste pas, bien qu'il y ait une certaine augmentation de l'azote non protéique. Par ailleurs, le taux du lactose reste pratiquement inchangé.

Pour ces raisons, la technique qui consiste à ajouter au lait destiné aux analyses, du formol et du trioxyméthylène nous paraît la plus recommandable. Ajoutons enfin qu'elle a l'avantage de ne pas mettre en œuvre un produit toxique.

\section{RÉFÉRENCES BIBLIOGRAPHIQUES}

[1] X. Rocques. La conservation des échantillons de lait destinés à l'analyse. Ann. Fals. Fraudes, 1912, 338.

[2] A. Kling, Gelin et Lassieur. Recherches sur l'altération des laits bichromatés. Ann. Fals. Fraudes, 1914, 244.

[3] L. Magnier de la Source. Observations sur la valeur du procédé officiel de conservation des laits par le bichromate. Ann. Fals. Fraudes, 1920, 550.

[4] M. Hinard. L'altération des laits bichromatés. Ann. Fats. Fraudes, 1913,233 et $1920,463$.

[5] Filaudeau, La conservation des échantillons de lait. Ann. Fals. Fraudes, 1923, 515.

[6] Letourneur, Hugon et Valin. Conservation des échantillons de lait par le formol et le trioxyméthylène. Ann. Fals. Fraudes, 1926, 544.

[7] G. Deniges. La conservation des échantillons de Iait destinés à l'analyse. Ann. Fals. Fraudes, 1912, 599.

[8] Fonzes-Diacon et LAForce. Le chromiforme : nouveau conservateur des laits d'expertise, Ann. Fals. Fraudes, 1928, 536.

[9] M. Voiret. Conservation des échantillons de lait en vue de leur analyse. Ann. Fals. Fraudes, 1951, 284.

[10] G. Guitonneau et $R$, Chevalier. Nouvelles techniques de préparation d'un milieu de culture à base de lait digéré. Le lait digéré par la papaïne. Le lait, 21, 1941, 1.

[11] A. Hirsch et E. Grinsted. Methods for the growth and enumeration of anaerobic spore formers from cheese, with observations on the effect of nisin. J, Dairy Res., 21, 1954, 101. 
[12] W. Dorner, P. Demont et D. Chavannes. Microbiologie laitière, $1945 ; 193$.

[13] I. Saenz Lascano Ruiz. Le dosage du lactose dans les laits altérés par fermentation lactique. Les eonditions d'hydrolyse quantitative du lactose dans un lactoserum. Ann. Fals. Fraudes, 1954, 167 et 285.

[14] S. J. RowLAND. The determination of the nitrogen distribution in milk. J. Dairy Res., 9, 1938, 42.

\title{
DES DANGERS QUE SEMBLE COMPORTER L'UTILISATION DE L'ALCOOL ISOAMYLIQUE DANS LES LABORATOIRES D'ANALYSES DE LAITERIES
}

\author{
par \\ RAPHAEL MOREAU
}

L'alcool isoamylique $\mathrm{CH}_{\mathrm{CH}^{3}}^{\mathrm{CH}^{3}}>\mathrm{CH}-\mathrm{CH}^{2}-\mathrm{CH}^{2} \mathrm{OH}$ est couramment employé dans les laboratoires de laiterie qui, pour la détermination de la teneur en matière grasse des laits, crèmes et fromages, utilisent la méthode butyrométrique "Gerber" ou des méthodes analogues.

Le rôle de cet alcool est de détruire l'émulsion de la matière grasse. Lors de chaque analyse un volume de $1 \mathrm{~cm}^{3}$ est introduit dans le butyromètre au moyen d'une ampoule, munie d'un robinet doseur.

La manipulation de cet alcool ne présentait jusqu'à ces derniers temps aucun danger en raison des faibles quantités mises en œuvre par suite du nombre relativement restreint des analyses effectuées journellement, en raison aussi de la nature de l'appareil mesureur employé.

La généralisation du paiement du lait suivant sa richesse en matière grasse et la disparition du beurre fermier ont augmenté, dans des proportions très importantes, le nombre quotidien des analyses effectuées dans les usines laitières (plusieurs centaines par jour et par usine au lieu de plusieurs dizaines). Cette situation nouvelle a incité certains constructeurs de matériel de laboratoire à concevoir des doseurs multiples permettant d'introduire l'automatisme dans l'application des méthodes butyrométriques et de réaliser ainsi des gains de temps importants.

Malheureusement, certains appareils doseurs à tubes plongeurs présentent, par suite de leur conception, le grave inconvénient de favoriser l'évaporation de l'alcool isoamylique dont les vapeurs se répandent dans l'atmosphère.

D'autre part, toutes les précautions ne sont pas toujours prises, lors des manipulations, pour éviter cette évaporation. Les personnes 